\title{
Reflexiones acerca de las necesidades de formación docente en Colombia en los tiempos de la sociedad líquida
}

\author{
López Rodríguez, Rubiela Rocío \\ Revista Educación, vol. 45, núm. 1, 2021 \\ Universidad de Costa Rica, Costa Rica \\ Disponible en: http://www.redalyc.org/articulo.oa?id=44064134031 \\ DOI: https://doi.org/10.15517/revedu.v45i1.42233
}

Reflexiones acerca de las necesidades de formación docente en Colombia en los tiempos de la sociedad líquida

\section{(c) $(1) \Theta$}

Esta obra está bajo una Licencia Creative Commons Atribución-NoComercial-SinDerivar 3.0 Internacional. 
Revisiones Bibliográficas

\title{
Reflexiones acerca de las necesidades de formación docente en Colombia en los tiempos de la sociedad líquida
}

\author{
Thoughts on Teacher Training Needs in Colombia in Times of Liquid Societies
}

Rubiela Rocio López Rodriguez

Universidad Nacional, Colombia

rlopez.IEMC@gmail.com

(iD https://orcid.org/0000-0003-2218-451X

\author{
DOI: https://doi.org/10.15517/revedu.v45i1.42233 \\ Redalyc: http://www.redalyc.org/articulo.oa?id=44064134031
}

Recepción: 21 Junio 2020

Aprobación: 02 Octubre 2020

\section{Resumen:}

El presente trabajo plantea la reflexión acerca de las diversas necesidades de formación del personal docente, teniendo en cuenta las dinámicas de sociedad líquida que se experimentan en la actualidad. En primer lugar, se hace referencia a la visión de los organismos internacionales con respecto a la importancia de la formación de educadores, las críticas al currículo impartido en las facultades de educación y la necesidad de replantear aspectos como el trabajo colaborativo y los objetivos institucionales, asumiéndolos como prioridades de formación. Por otra parte, se realiza una crítica a la formulación de nuevas políticas educativas en Colombia, que vulneran gravemente la profesionalización docente en nombre de la calidad educativa. Se elabora una recopilación de necesidades educativas para la sociedad de hoy, una sociedad marcada por las políticas neoliberales, el capitalismo salvaje y la destrucción de la vida. Finalmente, se proponen nuevas estrategias para la formación de las y los docentes y así, hacer frente a las nefastas consecuencias socioeconómicas y culturales derivadas de la modernidad líquida.

Palabras claVe: Formación docente, Política educativa, Sociedad líquida, Pedagogía, Humanismo.

\section{Abstract:}

Upon reflecting on teacher training needs from various perspectives, it is important to consider teacher training strategies required to confront the disastrous socio-economic and cultural consequences produced by liquid modernity. International organizations emphasize the importance of teacher education. Yet, the composition of curriculums for Education majors at universities in Colombia has many gaps and does not include collaborative work or institutional objectives within their teacher training priorities, for example. Many fallacies have also been detected when formulating new educational policies in Colombia, which are misconstrued as necessary to uphold educational quality, but which, actually, violate the professionalization of teachers. Given that today's society, characterized by neoliberal policies, savage capitalism and the destruction of life, faces specific educational needs. new strategies must be proposed to prepare teachers to face the nefarious economic and cultural consequences of liquid modernity. KeYwords: Teacher Training, Educational Policy, Liquid Society, Pedagogy, Humanism.

\section{INTRODUCCIÓN}

El personal docente es parte fundamental en los procesos de enseñanza, cada una de sus acciones en el aula ejercen una influencia directa en la construcción del conocimiento y sobre todo en la motivación o voluntad por aprender. La trascendental influencia de las maestras y maestros, hace que la política educativa se centre en brindar una formación integral, pertinente y actualizada; de esta manera, avanzar hacia una óptima calidad en los procesos educativos. La docencia como profesión exige diversas posturas éticas para desempeñar un rol de alta calidad; en primer lugar, es una profesión que demanda actualización pedagógica continua, pues los procesos educativos deben responder a las necesidades cambiantes de las comunidades; en segundo lugar, la formación docente debe presentar solidez en los temas pertinentes al área de conocimiento, pues la efectividad de la enseñanza implica un amplio dominio de los contenidos de la asignatura; finalmente, es necesario que la formación docente promueva la investigación - acción educativa, proyectos que permitan 
que las dinámicas escolares transformen la realidad de las comunidades, pues la misión del personal docente no solo influye en las y los estudiantes sino que también incide en la evolución de la sociedad. Según López y Granada (2007), quienes citan a Honoré:

La formación humana se centra desde la mutabilidad de la especie humana, esto apunta hacia la creación reflexiva más que a la reproducción técnica. La cultura es un escenario de investigación, práctica y convivencia de un sujeto que, al construir, se construye (p. 2).

Una acertada política de formación permite consolidar la profesionalización docente, esto implica incentivar el desarrollo de las cualidades del magisterio, su autonomía, creatividad, capacidad de respuesta a las demandas cognitivas y sociales actuales, capacidades académicas para la comprensión e investigación de los avances científicos relacionados a su propia área y el saber pedagógico. Los programas de formación docente pueden exponer la evolución de los modelos pedagógicos y la formulación de nuevos enfoques que inspiren al/la docente a redefinir su didáctica acorde con los avances en todos los campos de la ciencia.

El desafío más grande de las maestras y maestros en la actualidad es transformar una sociedad moderna fría, pragmática, individualista e insolidaria; una modernidad liquida, en donde los individuos de la clase trabajadora no presentan metas seguras u objetivos a largo plazo, sino más bien la incertidumbre de vivir el día a día, mientras las élites vivencian la extrema libertad que les da el libre mercado para infestar los medios con el marketing de sus empresas, expropiar territorios, extraer recursos naturales no renovables, manipular ideologías, manejar voluntades y mecanismos de justicia, todo en nombre de la globalización y el mejoramiento de su economía.

En el presente artículo se describen importantes factores a tener en cuenta en la política de formación docente. Se resalta el contraste entre los discursos del estado sobre las demandas de altos niveles de calidad educativa medidos por medio de evaluaciones estandarizadas. Por otra parte, se hace un análisis de la sociedad actual en los tiempos de la modernidad, los riesgos que corre la humanidad al seguir los ideales de una sociedad líquida, donde la perspectiva de estabilidad y futuro se desvanecen en un mar de inestabilidades y miedos, revisando contenidos que posibilitan un verdadero cambio social donde prevalezca la justicia, el bienestar individual y comunitario.

\section{VISIÓN GENERAL SOBRE LAS NECESIDADES DE FORMACIÓN DEL MAGISTERIO}

Diferentes organizaciones internacionales resaltan la trascendental importancia de los programas de formación de educadores como meta principal para alcanzar una alta calidad educativa en todos los niveles académicos; podría decirse que la formación docente es el eje principal en el desarrollo cultural y económico de la nación. Al respecto, De la Rosa, Giménez y De la Calle (2019) mencionan La Agenda 2030 y su meta:

La educación se enfrenta al reto de actualizar la sociedad con nuevos conocimientos fortaleciendo los valores humanos y democráticos, la necesidad de promover el desarrollo sostenible mediante sus procesos y la promoción de una cultura de paz, la ciudadanía mundial y la contribución de la cultura al desarrollo sostenible (p. 182).

Dicha reflexión se deriva en debates acerca de la calidad educativa, la profesionalización docente, la actualización de la práctica educativa y los planes de estudio de las facultades de educación. Una interpretación equivocada de calidad educativa tiende a considerar, como única evidencia de calidad, los resultados de las evaluaciones estandarizadas y tiende, además, a responsabilizar en exclusiva al desempeño docente de los bajos resultados de las y los estudiantes en dichas evaluaciones. Este fenómeno hace que los programas de capacitación docente tengan como meta una formación encaminada únicamente a elevar los puntajes en pruebas estandarizadas, para el contexto colombiano, las denominadas pruebas Saber, que miden conocimientos en áreas específicas. 
Teniendo en cuenta que una educación de calidad no se centra solo en resultados de evaluaciones de conocimientos, es pertinente entender la calidad educativa como una oportunidad crucial para que las y los estudiantes accedan a oportunidades académicas y laborales, a través del desarrollo de competencias y el logro de desempeños diversos. En palabras de Corredor (2019):

Las políticas educativas para el mejoramiento de la calidad de la educación media en Colombia presentan múltiples retos, por un lado, los temas de cobertura, acceso y permanencia y por otro lado los temas relacionados con el desarrollo de competencias y los procesos de enseñanza-aprendizaje. Considerar políticas educativas enfocadas a la cobertura, acceso y permanencia únicamente supone una visión limitada de la calidad de la educación (p. 122).

Las críticas acerca de los programas de formación para docentes enfocados en el mejoramiento de resultados en evaluaciones estandarizadas, contemplan múltiples aspectos. En investigaciones realizadas por Álvarez (2015), se especifican los siguientes factores como debilidades del currículo de formación:

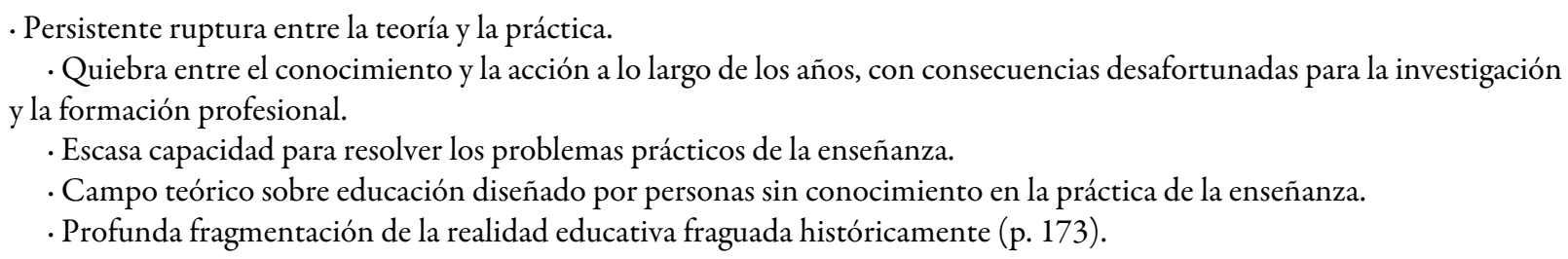

El dilema entre capacitación o formación se da en el marco de grandes inversiones que no reflejan los resultados esperados; asimismo, los programas de formación impartidos por instituciones sin acreditación en alta calidad, valoración que otorga el Consejo Nacional de Acreditación de Colombia, orientan bajo los mismos modelos pedagógicos tradicionales de décadas anteriores.

La publicidad que se hace de los rankings de centros educativos acrecienta la falta de credibilidad en el desempeño docente, lo que hace necesario establecer programas de capacitación e investigación permanentes con los que se demuestren sus capacidades y se eleve su estatus cultural. Por otra parte, las y los docentes de instituciones oficiales son los empleados públicos con menor remuneración económica, esto hace que sea una profesión indeseable para muchos jóvenes que no ven en la docencia una buena oportunidad laboral. Los escasos salarios de las y los docentes también hacen que sea imposible el pago y mantenimiento en algún programa de posgrado, pues sus altos costos solo pueden ser cubiertos por alguna de las reducidas becas que se puedan ofrecer. Es necesario que se analicen los diversos factores que obstaculizan la transformación de la escuela, ya que el impacto positivo que puede lograr el profesorado en el aula se ve obstaculizado por la resistencia que le ofrecen dichos factores. En palabras de Camargo et al. (2004):

Las críticas acerca de los programas de formación para docentes enfocados en el mejoramiento de resultados en evaluaciones estandarizadas, contemplan múltiples aspectos. En investigaciones realizadas por Álvarez (2015), se especifican los siguientes factores como debilidades del currículo de formación:

Los esfuerzos de formación del personal docente, tanto inicial como permanente realizados hasta ahora, no han dado lugar
a un mejoramiento de su estatus laboral y profesional, ni han impactado su reconocimiento social, ni contribuido a la
construcción cualificada de su oficio como profesional. Se aduce como posible explicación la dificultad para contrarrestar la
fuerte influencia de otras variables, como el origen social de los docentes. Ello ha llevado a que se considere que 'la educación
es una actividad donde la profesionalización integral no sería posible ni conveniente', pues 'una cantidad significativa de
decisiones que el docente tiene que asumir están basadas en opciones éticas, determinaciones culturales, apreciaciones
subjetivas y teorías de escasa corroboración empírica'. Desde este punto de vista, sería posible postular de manera favorable
una hipótesis basada en la desprofesionalización (p. 85).

La capacitación docente también influye de manera positiva en el desarrollo y cualificación de las instituciones educativas. Una cultura académica proporciona un clima de valores, autosuperación, trabajo en equipo, compromiso institucional. Estos logros individuales se reflejan posteriormente en logros institucionales, distribución equitativa de recursos, definición de metas institucionales; redefinición de 
modelos de organización, desarrollo de programas de gestión y espacios de evaluación y mejoramiento institucional. Álvarez (2015) resalta que:

Es importante para las instituciones educativas contar con una eficiente cultura organizacional, pues es la hoja de ruta para implementar para aplicar las estrategias que les permitan lograr sus objetivos desde el punto de vista estructural, metodológico, pedagógico y conductual (p. 21).

La capacitación docente debe promover no solo el aprendizaje para alcanzar metas individuales, sino que debe impulsar el logro de los objetivos y metas institucionales, de ese modo las instituciones educativas avanzan hacia los propósitos institucionales y hacia la transformación del centro educativo en una comunidad de aprendizaje dinámico, conjunto e interrelacionado. Al respecto, Pérez y Coutín (2005), refieren las definiciones de gestión del conocimiento como:

La gestión del entorno optimiza el conocimiento. El conocimiento en sí mismo no se puede gestionar. Gestionar el entorno es hacer que la gente lea, piense y coordine entre equipos. El proceso de desarrollar, estructurar y mantener la información, de transformarla en un activo crítico y disponerla a una comunidad de usuarios, definida con la seguridad necesaria. Esta incluye el aprendizaje, la información, las aptitudes y la experiencia desarrolladas. Los cambios de mercado exigen una producción flexible y una rápida capacidad de respuesta a las demandas no previstas. Cuando se habla de trabajo en equipos se refiere al trabajo en grupo o dinámica de grupo, como también se le conoce (p. 36).

El trabajo en equipo como centro de la capacitación docente para el logro de los objetivos institucionales es necesario en los programas de formación del magisterio. Los profesionales de la educación representan y trabajan por la identidad de la institución donde laboran, encarnando su visión, misión y objetivos; por otra parte, son modelos de liderazgo, bien sea en temas de gestión, administración o liderazgo pedagógico; son agentes promotores del trabajo colaborativo en su institución. Los profesionales de la educación son generadores de espacios de reflexión, evaluación y crítica de los procesos administrativos y educativos que se llevan a cabo en el centro educativo; asimismo, formulan planes de mejoramiento institucional continuo. Promover el trabajo en equipo es un componente fundamental para el crecimiento de los procesos de enseñanza, la coevaluación y la contribución individual y colectiva en los proyectos institucionales son una proyección de las metas individuales y la motivación a la consecución de logros académicos por parte de nuevos/as profesionales docentes. Es importante resaltar que "el intercambio de ideas, estrategias, resolución de problemas, materiales, posibilita el aprendizaje entre pares académicos e inspira hacia la profesionalización de la labor docente" (Sánchez y Galiano, 2019, p. 107).

\section{REFLEXIONES SOBRE LA POLÍTICA EDUCATIVA COLOMBIANA}

En nombre del mejoramiento de la calidad educativa y en busca de una mayor eficiencia de los centros educativos estatales, se han realizado una serie de reformas en las políticas educativas promovidas por el Ministerio de Educación Nacional de Colombia (MEN). Dichas reformas han generado una enorme resistencia, ya que son reformas que no se conciertan con las comunidades educativas y que lesionan gravemente la profesionalización docente. Numerosos movimientos sindicales alegan que las reformas educativas representan un retroceso enorme en la lucha por reivindicar la labor docente. En palabras de Flórez (2017) "en el aspecto pedagógico, las reformas curriculares comparten comunes denominadores como la irrupción de conocimientos prácticos y el desprendimiento de fundamentos teóricos y humanísticos” (p. 8).

Una de dichas leyes es el Estatuto de Profesionalización Docente (Ley No715, Decreto No1278) (MEN, 2002), que recorta los derechos de carrera docente; asimismo, desaparece el régimen especial, la estabilidad laboral, el derecho a la formación permanente ofrecida por el Estado, obstaculiza los procesos de ascenso, recorta los derechos sindicales y políticos, desconoce los derechos de los profesionales de la educación de instituciones privadas, se establecen procedimientos de evaluación punitiva para docentes sujeta a criterios 
subjetivos y permite que profesionales sin formación pedagógica ingresen a la carrera docente, su formación pedagógica se reduce a un curso de seis meses de duración.

Como crítica al sistema meritocrático de ingreso a la carrera docente, se podría decir que no basta con el conocimiento disciplinar o profesional, ya que la labor educativa es mucho más compleja que solo transmitir conocimiento. La efectividad de los programas de formación docente depende de la formulación de planes de estudio con énfasis en saberes para la vida, dirigidos hacia la comunidad educativa y la construcción de saber con sentido social, en lugar de enseñar exclusivamente para las evaluaciones externas. Al respecto Lucio (2019) afirma:

Superar el modelo actual de la formación docente y de la educación correspondiente, demanda remontar esta violencia simbólica naturalizada y demanda una praxis en la formación docente, capaz de 'superar el miedo a la libertad'. La formación docente transformada y transformadora, requiere la libertad de palabra y de autonomía. Demanda la liberación de la expresión y de la investigación científica, acompañadas y alentadas por un espíritu crítico y ético, para construir sociedades del conocimiento (p. 34).

Actualmente no se cuenta con mecanismos de diálogo que posibiliten la reflexión y la toma de conciencia en torno al reconocimiento del rol social que desempeña la profesión docente. Los profesionales de la educación, que actualmente se sienten obligados a transmitir trozos de conocimientos sin relación, reclaman aportar en la construcción de saber universal y local, lo cual es fundamental en el fortalecimiento de la identidad y la transmisión de la cultura. Es importante resaltar la investigación de Valencia (2016), en la que afirma que:

Una debilidad en la formación de los docentes en Colombia es la escasa investigación, pues los grupos de investigación de las facultades de educación son muy pocos y la clasificación de los existentes es muy baja, no se destinan recursos para investigar en dicho campo en el país; resalta también la dificultad de construir una comunidad académica debido a la provisionalidad de la planta de docentes. A esto se le suma la cultura de la evaluación mencionada anteriormente (p. 9).

\section{Riesgos DE UNA SOCIEDAD LÍQUIDA}

Diversas investigaciones establecen los nuevos desafíos de la sociedad frente a la sociedad líquida. Avellaneda (2015), establece que la educación no se limita sólo a aumentar los conocimientos, sino que también desarrolla habilidades, actitudes y capacidades en las que se tiene que destacar el cuidado del medio ambiente y el buen uso de los recursos naturales. En otro estudio, Camusso (2019) revisa las características de la sociedad líquida y propone diferentes estrategias didácticas para motivar a las y los estudiantes. Por último, Canaza (2018) reflexiona acerca del espejismo de las redes sociales en la modernidad líquida, cómo altera la conducta individual de los jóvenes y a su vez, altera la estructura política, económica, social y cultural.

Zygmunt Bauman (2003), describe la avanzada descomposición de las relaciones sociales actuales como consecuencia de la apertura de mercados en una dinámica sumamente consumista y capitalista. El símil que representa los estados de la materia y las concepciones sociales ilustra la transitoriedad de los valores y actitudes humanas, el individualismo, egoísmo, la competencia salvaje de un individuo que debe adaptarse constantemente al cambio, sin oponer resistencia para sobrevivir las olas de la modernidad. Así como existieron sólidos valores morales y propósitos en las comunidades; la modernidad líquida es una figura que ilustra el constante cambio actual, así como los líquidos deben adaptarse al recipiente que los contenga, las relaciones humanas deben ceder a las fuerzas del sistema que las rige, de esta manera obtener el éxito y sobresalir.

Esta idea de flexibilidad y constante cambio destruyó el ideal de estabilidad y la concepción de certeza. Por ende, los sujetos, que en apariencia son libres, dependen permanentemente del sistema que los contiene, la cultura laboral y familiar ya no conciben estabilidad, de esta manera, los individuos se encuentran a la deriva sin una visión clara del futuro, abandonándose al bienestar inmediato del día a día. En palabras de Bauman (2003): 
La modernidad líquida, cuyo eje ya no es la ética del trabajo sino la estética del consumo ha suplantado las estructuras institucionales de la etapa anterior, que proporcionaban seguridad y confianza en el futuro, y las ha reemplazado por otras mucho más transitorias y menos sólidas, y por ello incapaces de orientar y dar sentido a largo plazo a la vida individual y colectiva. Los individuos son invitados a estar continuamente en movimiento sin la esperanza de consolidar ninguna posición. 'El único propósito de estar en movimiento es permanecer en movimiento'. Todo ello hace que la experiencia humana carezca de continuidad histórica, y por ello también de una perspectiva arraigada profundamente en el tiempo que le dé sentido. Es más, dicha continuidad queda desacreditada en una cultura del cambio en la que el pasado ya no cuenta, sino para ser refutado en nombre de un presente que se actualiza permanentemente (p. 169).

En vista que todo este escenario crea una inmensa incertidumbre, los individuos interiorizan el miedo a resistirse al cambio continuo en su ritmo de vida, de acuerdo con López, Restrepo y López (2013), "la necesidad de vencer la inercia y el miedo de fracasar que cunde en los mandos medios de muchas organizaciones" (p. 155); sin embargo, lo que si permanece muy sólido es el sistema económico al que toda persona debe sujetarse. En función de dicha fluctuación de mercado, cambio de perfil, funcionalidad, exigencias de capital, los individuos deben negarse a sí mismos para satisfacer las metas, resultados u objetivos esperados, incluso causando grandes episodios de estrés, en palabras de González (2014), "los miembros de las organizaciones trabajan eficazmente para alcanzar metas comunes, sujeto a las reglas básicas establecidas con anterioridad, lo cual puede provocar estrés si no es cumplido eficazmente” (p. 13). También sigue muy firme la división de clases, que se muestra arraigada, incluso preestablecida; mientras que las personas en sus diferentes roles personales o laborales son fácilmente reemplazables.

Con respecto al estudio de Bauman (2003), en el mundo de la posmodernidad se juzga, evalúa y valora a un individuo según su relación al consumo de productos. La sociedad de consumo está completamente desregulada y persigue cada día algo novedoso como meta de realización personal. Se les persuade para que el consumo constante sea su prioridad, conllevando a las personas a diversas inseguridades y miedos, lo que los frustra para seguir consumiendo en un círculo vicioso del que se beneficia la economía, mientras el planeta sufre, ya que día a día pierden más recursos vitales. En una sociedad que no solo sobrevalora el hoy, sino que no espera con mucha expectativa el mañana, se debe entrar en la dinámica económica antes de ser excluido socialmente por ser diferente al no poseer o presumir bienes de consumo. En este propósito de producción en masa llama la atención un fenómeno denominado obsolescencia programada, que, según Hindle (2008), es una:

Estrategia de manufactura implementada por las empresas para limitar la vida útil de los productos que fabrica, generando una expiración controlada en virtud de la cual dichos productos pasarán a ser inservibles y/o inútiles en una fecha conocida y planificada (p. 147).

Reduciendo el periodo de vida útil de un producto, se procede a convencer al individuo de adquirir uno nuevo permanentemente, generando más ganancias para las empresas y una eterna dependencia del consumidor. Siguiendo lo que señala Yang (2016):

En cuanto a la obsolescencia programada, se intenta sustituir un producto existente en el mercado por otro más nuevo.
Los ejemplos más claros son los libros de texto en la educación y la actualización de hardware y software en la industria
tecnológica. La tarea más dura para el fabricante es la de convencer al consumidor para que adquiera el nuevo modelo
para sustituir el que tiene desde hace poco. El entorno competitivo hace que esta tarea sea más fácil ya que el consumidor
considera que su estatus social puede verse afectado si no tiene el último modelo del producto. Por esta razón, los fabricantes
juegan muchas veces con las ventajas competitivas de los consumidores. En realidad, frecuentemente este último modelo no
tiene cambios significativos, es más, sus cambios suelen ser más de aspecto estético que de aspecto funcional. Pese a ello el
consumidor cae en la trampa igualmente y adquiere el último modelo (p. 7 ).

La publicidad juega un papel importante en el mantenimiento de ese circuito diabólico, Latouche (2009) indica que, "son necesarios tres ingredientes: la publicidad, que genera el deseo en el consumidor; el crédito, que le provee los medios; y la obsolescencia acelerada y programada de los productos, lo cual renueva su necesidad" (p. 17). El mercado de la publicidad crea necesidades donde realmente no existen, llevando a los 
consumidores a no ser conscientes de sus necesidades prioritarias, los cálculos acerca del mercado de oferta y demanda de productos se apoyan en el marketing para aumentar los ingresos. La corta vida útil de los artefactos es similar a la corta vida útil de las personas que también son pensadas como cifras, números y estadísticas. Por otra parte, es imposible negar la injusticia de la constante urgencia por sobrevivir de los sujetos de clase media - baja en la sociedad moderna, la incertidumbre de vivir el día a día sin tener nada fijo, no solo se traduce en las superfluas e insensibles relaciones personales y laborales, sino en individualismo, egoísmo, competencia desleal, corrupción, indiferencia y pobreza. Hofstede (2011) formula que:

Para comprender mejor las tendencias humanas en la sociedad líquida, se examinan seis dimensiones que describen el comportamiento cultural de tres países y sus tendencias, analizando la distancia al poder, que representa el autoritarismo; el nivel de individualismo, la prevalencia de la masculinidad, el miedo a la incertidumbre, la orientación al largo plazo y la indulgencia que describe la libertad de satisfacción de necesidades (p. 5) (Ver Figura 1).

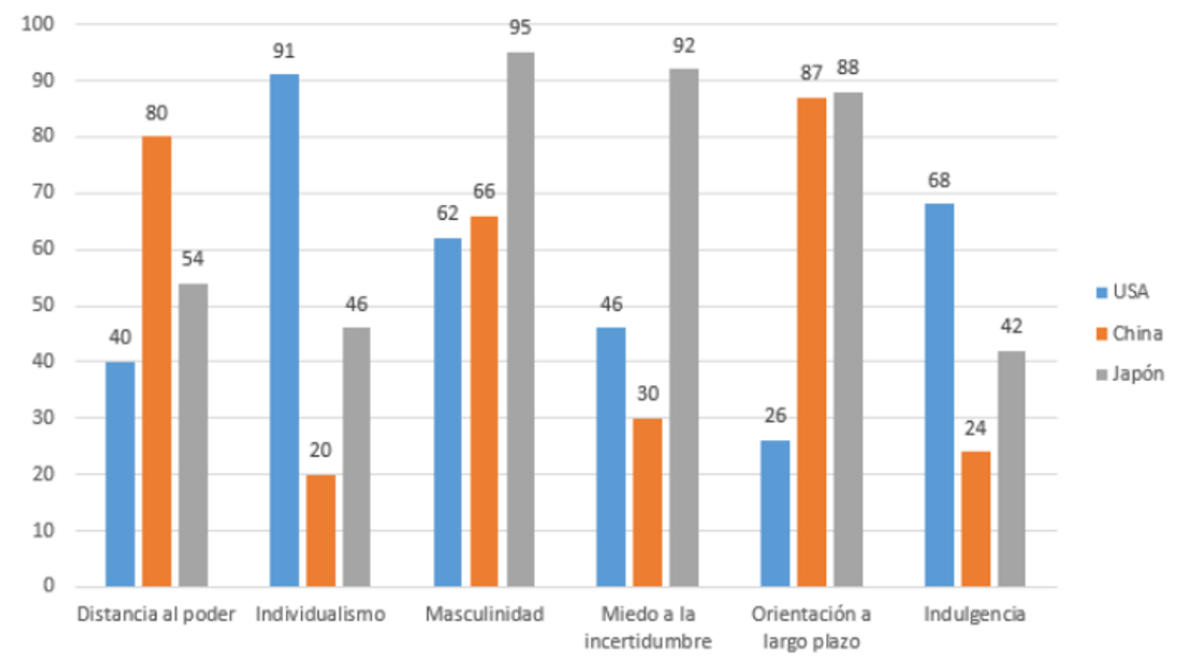

FIGURA 1

Dimensiones de Hofstede para Estados Unidos, China y Japón Hofstede, G. (2011). Sitio web Hofstede Insights.

Del mismo modo en el que la modernidad líquida es un ancla constante a la esclavitud laboral moderna de la clase media - baja trabajadora, para la elite social representa libertad plena de acción, autonomía y realización personal. Las dos realidades anteriores degradan y afectan negativamente a largo plazo todas las instituciones y planos de vida de las personas, sobre todo en los conceptos interiorizados de familia, derechos humanos, ética y valores. Este proceso de desvinculación de todos los arraigos culturales anteriores despoja al individuo de identidad propia, así que termina por seguir modelos estereotipados como su ideal. Lo opuesto a liquidez de ideales, son conceptos como: apoyo, soporte, sentido y estabilidad, los cuales significan una amenaza para el estilo de vida que se lleva. Tampoco cobra sentido la función de los centros educativos; debido a eso, se produce una profunda crisis del sistema, ya que este se basa en el proyecto de preparar para la vida a largo plazo; sin embargo, al término de los ciclos de enseñanza, los contenidos ya son obsoletos. Corroborando lo anterior, el análisis de las dimensiones de Hofstede para Colombia se observa en la figura 2: 


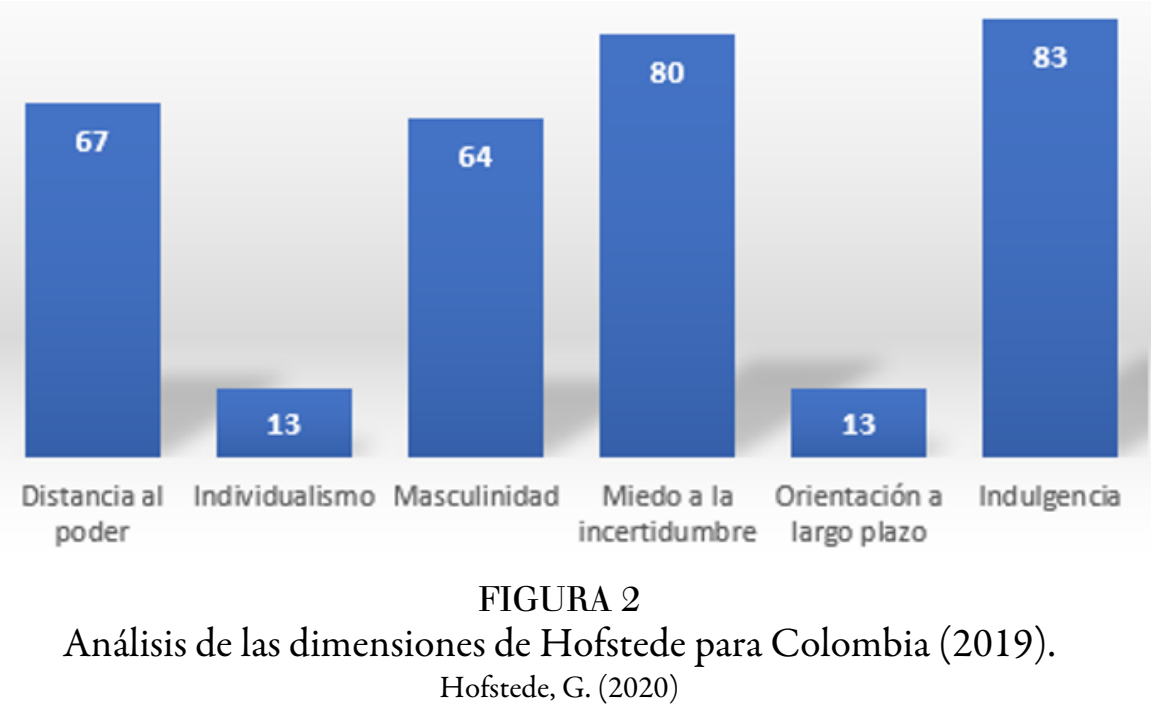

La sensación de no poseer nada estable, se traduce también en los vínculos y las relaciones. Con ayuda de las nuevas tecnologías de la información y la comunicación, la cual posibilita una interconectividad instantánea, se proporciona la ilusión de compañía; pero a pesar de dicha sensación, las relaciones personales que son instantáneas, también se deshacen instantáneamente, así que se convierten en relaciones solubles, breves y frágiles. Es impresionante la capacidad ambivalente de la teoría de la modernidad líquida; por un lado, enuncia y promociona libertades mientras que, por el otro, es la representación de la dependencia en todo sentido. Los fenómenos sociales que se analizan de esta visión líquida de modernidad se muestran como conductas generalmente individualistas, esto impide la unión en movimientos en torno a ideales de beneficio común, que son absolutamente necesarios teniendo en cuenta el bienestar de los sujetos; de esta manera, se extinguen los movimientos sociales al no contar con el respaldo democrático para su consecución.

La ausencia de conciencia social, de memoria histórica, de proyecto de vida, transmite desazón y desesperanza; estos son solo algunos de los problemas que dividen aún más a los individuos. En palabras de Bauman (2007):

En esto consiste la libertad proclamada por la sociedad moderna líquida. Pero se trata en realidad de una libertad sin opciones, huérfana, porque los individuos están siempre obligados a elegir, y porque, además, los que eligen no disponen de los instrumentos institucionales necesarios para controlar sus propias elecciones, lo cual crea todavía más inseguridad e incertidumbre (p. 104).

\section{Metodología}

El siguiente trabajo es una revisión bibliográfica en la que, en primer lugar, se revisan investigaciones centradas en las necesidades actuales de formación de docentes, la reflexión sobre la concepción sesgada de calidad educativa, de qué manera el mejoramiento de la calidad educativa implica un análisis multidimensional donde prevalece la necesidad de una formación que prime el trabajo colaborativo y la gestión del conocimiento. Desde ese punto de vista se puntualizan las falencias de la norma que rige la actividad docente colombiana actual. Como referencia de este trabajo se recoge la visión de Zygmunt Bauman, que describe las características de la sociedad líquida actual, la liquidez de las relaciones sociales y las consecuencias del consumismo, la globalización y el neoliberalismo. Se tomó la decisión de recuperar los aportes de autores como el mencionado Bauman (2003), Honoré (1980), González (2014), e investigaciones en pedagogía y sociedad líquida de Álvarez (2015) y Camusso (2019). Por último, se retoman los aportes de De Zubiría 
(2013) en el planteamiento de una formación humanista integral y diversificada para el magisterio, ya que propone una metodología basada en la flexibilización curricular, la formación en el pensar, hacer y amar.

\section{ESTRATEGIAS DE FORMACIÓN DOCENTE ENFOCADA EN EL CAMBIO Y LA JUSTICIA SOCIAL}

La utopía de detener el curso de estos tiempos oscuros encuentra su esperanza en una educación con una nueva visión. Para esto, la sociedad en general y la comunidad educativa en particular, debe vencer el miedo. Siguiendo a Bauman (2007), la incertidumbre se convierte en un miedo constante con el que conviven todos los individuos, miedo a la no aceptación, a la exclusión, al poseer y al no poseer, miedo al miedo mismo. Son miedos reales, irracionales o infundados, que sirven para manipular la consciencia de las personas, sin un origen específico y que al ser difusos son difíciles de combatir. Vencer el miedo a vender la dignidad humana para sobrevivir o sobresalir, a oponerse a un sistema esclavizante, al cambio de paradigmas, es el primer paso para la transformación de las escuelas.

Debido a la liquidez de los principios, el proceso de enseñanza perdió todo su significado. La crisis de contenidos, que en un principio promovían el progreso y la justicia, han perdido toda justificación. El ideal de los tiempos posmodernos es el cambio, no la acumulación de saberes; en especial, si no se relacionan a movilidad constante, lo que recrea una amnesia continua y dinámicas de desaprendizaje periódico. La nueva educación no puede ser un instrumento para el afianzamiento de la dinámica líquida; por el contrario, por medio de la educación se debe encontrar la manera de volver a la solidez de los derechos y la dignidad humana. Para Bauman (2007), "La educación es víctima de la modernidad líquida, educar es hacer una inversión en los próximos cien años” (p. 133). No es posible negar la realidad en la que vivimos; sin embargo, por medio de la educación, se alcanzaría un elevado estado de consciencia de los sujetos, un estado permanente en el que se analice el problema de la fluidez, transformando los ideales individualistas en anhelos de trascendencia personal y colectiva.

La producción en masa y la economía extractiva que afecta en especial a los países tercermundistas, América del sur y África, es una de las causas de la extrema pobreza y desigualdad del planeta tierra. Dichos efectos lograrían disminuirse si la política gubernamental global y las decisiones en política educativa revisaran sus prioridades por el bien de las futuras generaciones. Al querer transformar la funcionalidad de los sistemas educativos, es prioritario resaltar la importancia de la educación humanista - ambientalista, en el marco de una educación para la vida y para la superación personal en ideales y valores, en armonía con la naturaleza que sustenta la vida de todos los seres de la tierra. Además de la concienciación en torno a los fines educativos, es necesario revisar los contenidos impartidos y aquellos contenidos que posibilitan la transformación social si son orientados en los currículos de licenciaturas en pedagogía.

En el marco de una educación para la vida y para la superación personal en el marco de los ideales y valores, en armonía con la naturaleza, De Zubiría (2013) plantea los nuevos desafíos de la educación en el siglo XXI como la necesidad de reconocer que el ser humano presenta diversas dimensiones:

La primera dimensión está ligada con el pensamiento; la segunda con el lenguaje; la tercera con la interacción; la cuarta, con el afecto, la sociabilidad y los sentimientos; y la última, con la praxis y la acción. En un lenguaje cotidiano, diríamos que el ser humano piensa, se comunica, interactúa, ama y actúa; y que es obligación de la escuela enseñarnos a pensar mejor, amar mejor y actuar e interactuar mejor (p. 7). (Ver Tabla 1). 
TABLA 1

Transversalización de contenidos que desarrollan las diversas dimensiones humanas

\begin{tabular}{|c|c|}
\hline Saber y Saber Hacer & Saber ser \\
\hline $\begin{array}{l}\text { Respeto, solidaridad, valores, dignidad y } \\
\text { Sucesos históricos y ciencias }\end{array}$ & $\begin{array}{l}\text { - Inteligencia } \\
\text { emocional - }\end{array}$ \\
\hline políticas - $\quad$ Filosofía y pensamiento crítico - $\quad$ Sociología & Pensar, amar y actuar. \\
\hline de Ciencias Naturales - Ciencia, creatividad e & Formar para \\
\hline innovación. - $\quad$ Seguridad alimentaria y nutrición - & la vida. - \\
\hline Sustentabilidad y medio ambiente - $\quad$ Trabajo en equipo Area de & \\
\hline $\begin{array}{lc}\text { Humanidades - } & \text { Comunicación asertiva, investigación, debate y } \\
\text { argumentación. - } & \text { Pensar, comunicarse y convivir. - }\end{array}$ & \\
\hline $\begin{array}{l}\text { Manejo e interpretación de los medios masivos de comunicación (Radio, } \\
\text { televisión, redes sociales) Area de Matemáticas - }\end{array}$ & \\
\hline $\begin{array}{lc}\text { - } & \text { Educación financiera Area de Tecnología - } \\
\text { proactivos de las nuevas tecnologías - } & \text { Búsqueda, selección y uso de } \\
\text { la información. Area Artística - } & \text { Salud, ejercicio y rendimiento físico. }\end{array}$ & \\
\hline
\end{tabular}

Elaboración propia basada en De Zubiría (2013)

El autor señala que el estudiantado no debe asistir para aprender, sino para desarrollarse. La división por asignaturas debe propender a la transversalización de los saberes que pueden aplicarse en la resolución de los desafíos diarios de los ciudadanos. El saber cognitivo no es un saber válido si es exclusivamente memorístico y si no aporta en nada a la comunidad que rodea la escuela; esto quiere decir que el saber cobra validez cuando tiene un propósito de acción, con base en esta premisa se deben plantear los currículos de todos los niveles académicos, básica primaria, secundaria y formación profesional.

El objetivo de la educación es desarrollar los talentos de las y los estudiantes en aspectos cognitivos, valorativos, afectivos y prácticos. Esto requiere renovar el currículo, replantear profundamente la formación de docentes y cambiar la finalidad educativa; por tanto, cambiar la educación implica erradicar la cultura de la liquidez, resistirse y oponerse a ella de una manera férrea, pues es el camino más viable para salvar a la humanidad y a la naturaleza. Replantear los modelos pedagógicos que imperan en la actualidad implica establecer el principio de la autonomía. En palabras de Sanabria (2017), citando a Kant, la educación propicia la autonomía, la dignidad de un ser racional que no obedece a ninguna otra ley que aquella que él se da a sí mismo. En la actualidad, la sociedad tiende a doblegar la dignidad humana, desnaturalizar los propósitos del aprendizaje y llevar a los individuos al aislamiento y a la completa insensibilidad.

El currículo y planes de estudio de los centros educativos públicos en todos los niveles de Latinoamérica, tanto de básica primaria, secundaria y profesional, se caracterizan por su rigidez cognitiva, por su modelo pedagógico, didáctico y metodología de evaluación memorística, sin recursos tecnológicos; en adición a esto, se encuentra fragmentado en diversas asignaturas que no se integran y que no tienen ninguna función social práctica, un currículo discriminatorio que causa la liquidez de los ideales y la reproducción de los problemas sociales que padece la sociedad actual. En palabras de Miguel De Zubiría (2005):

Todas las asignaturas deben consolidar la lectura, la escritura, la escucha, el pensamiento, el conocimiento y la comprensión de sí mismo y de los otros. Por ello son competencias transversales. Lo anterior implica que, en sociales, en matemáticas, en artes, en lenguaje y en ciencias, la tarea central de la educación básica debería ser enseñar a pensar, comunicarse y convivir. Es así de sencillo y de claro. Sólo así garantizaremos un lineamiento curricular más pertinente, más contextualizado y menos rutinario que el actual. Sólo así garantizaremos el trabajo en equipo necesario para consolidar la calidad (p. 84).

No solo basta con mejorar la calidad dentro de la institución educativa, sino que se debe entender como obligatorio compartir el saber y los procesos educativos con las comunidades que los rodean, para esto todos los profesionales deben formarse de manera integral, para transformar la sociedad a la que se les ha condenado. 


\section{Conclusiones}

La formación de docentes es esencial en el desarrollo de los sujetos y de la sociedad, se les responsabiliza de los resultados académicos de las instituciones públicas, resultados asociados directamente a la calidad de los procesos de enseñanza. El profesorado es el actor más importante de la comunidad educativa, ya que domina su área de especialización, reflexiona dicho saber y critica la propia acción pedagógica, construyendo el conocimiento y motivando al estudiantado hacia el desarrollo de su autonomía, libertad y consciencia social.

Es imperante no solo reflexionar en torno a los programas de formación de docentes, sino diseñar estrategias educativas y de formación de profesionales que impliquen la participación de las escuelas en las comunidades por medio de saberes que las transformen. Urge educar para el futuro y volver a lo básico, retomar los sólidos valores de la democracia, del humanismo y del ambientalismo, para darle de nuevo sentido a la acción de educar. Este ideal representa no solo la única vía de esperanza de cambio y justicia social, sino la única manera de reconciliar la sociedad y la academia.

Las y los docentes en Colombia reclaman retomar el pacto social por la educación y retoman sus demandas en torno a la importancia del desarrollo profesional, dignificación, profesionalización y calidad de vida, formación y desarrollo profesional, investigación y acción en la formación universitaria, identidad profesional y gestión del conocimiento. Esto exige replantear la política pública educativa y los sistemas de formación y promoción docente en el país.

Es posible formar a las y los docentes en toda su diversidad e integralidad, diferenciando el aprendizaje conceptual del desarrollo humano. Por consiguiente, la posibilidad que la flexibilización curricular en la formación docente abrirá nuevas posibilidades de formación autónoma, ética y responsable. Derivado de los nuevos contenidos curriculares se favorece la solidaridad y la identidad cultural, contrario a la tendencia individualista que predomina actualmente. Es interesante pensar por qué, si todos los aspectos de la vida están sujetos a cambios constantes, los programas de formación se plantean de manera similar constantemente. Si sobrevalorar lo cognitivo ha llevado a la sociedad a su estado actual, la misión del/la docente es iniciar su renovación.

\section{ReFERENCIAS BibLIOgRÁFICAS}

Álvarez, C. (2015). Teoría frente a práctica educativa: algunos problemas y propuestas de solución. Perfiles educativos, 37(148), 172-190. Recuperado de https://n9.cl/6avbl

Avellaneda, V. (2015). El hombre de la sociedad líquida y los desafios de la educación actual. (Tesis de pregrado). Universidad Santo Tomás, Colombia. Recuperado de https://n9.cl/7i8t

Bauman, Z. (2003). Modernidad líquida. México: Fondo de cultura económica.

Bauman, Z. (2007). Vida de consumo. Madrid, España: CE.

Camargo, M.; Calvo, G.; Franco, M.; Vergara, M.; Londoño, S.; Zapata, F.; y Garavito, C. (2004). Las necesidades de formación permanente del docente. Educación y Educadores, (7), 79-112. Recuperado de https://www.redalyc .org/pdf/834/83400708.pdf

Camusso, P. (2019). ¿Cuáles son algunas de las características de la modernidad líquida que ponen en tensión cómo seducir a los estudiantes? Revista Voces de la Educación, 4(8), 17-27. Recuperado de https://n9.cl/ji8y

Canaza, F. (2018). La sociedad 2.0 y el espejismo de las redes sociales en la modernidad líquida. Revista In Crescendo, 9(2), 221-247. Recuperado de https://n9.cl/4wbt

Corredor, N. (2019). Factores de la calidad educativa desde una perspectiva multidimensional: análisis en siete regiones de Colombia. Plumilla Educativa, 23(1), 121-139. doi: https://doi.org/10.30554/plumillaedu.1.3350.2019 
De la Rosa, D; Giménez, P; y De la Calle, C. (2019). Educación para el desarrollo sostenible: el papel de la universidad en la agenda 2030. Transformación y diseño de nuevos entornos de aprendizaje. Revista Prisma Social, (25), 179-202. Recuperado de https://revistaprismasocial.es/article/view/2709

De Zubiría, M. (2005). Gestión de aula. Experiencia del proyecto de mejoramiento. Proceso de enseñanza - aprendizaje. Matemáticas y castellano básica primaria en cinco escuelas del departamento del Atlántico. Barranquilla: Fundación Promigas.

De Zubiría, J. (2013). El maestro y los desafíos a la educación en el siglo XXI. Editorial Revista Redipe, 825, 1-17 Recuperado de http://www.uasb.edu.ec/UserFiles/385/File/redipe_De\%20Zubiria.pdf

Flórez, J. (2017). La política pública de educación en Colombia y Estados Unidos: la irrupción de las reformas educativas globales en el caso colombiano y estadounidense. Revista Via Iuris, (23), 0-32. Recuperado de https: //www.redalyc.org/articulo.oa?id=273957284010

González, M. (2014). Estrés y desempeño laboral. Estudio realizado en serviteca altense de quetzaltenango. (tesis de pregrado). Universidad Rafael Landivar, Quetzaltenango, Guatemala.

Hindle, T. (2008). Guide to Management Ideas and Gurus. London: Profile Books Ltd \& the Economist.

Hofstede, G. (2011). Dimensionalizing Cultures: The Hofstede Model in Context. Online Readings in Psychology and Culture, 2(1). doi: https://doi.org/10.9707/2307-0919.1014

Hofstede, G. (2020). What about Colombia?. Hofstede Insights. Recuperado de https://www.hofstede-insights.com/ country/colombia/

Honoré, B. (1980). Para una teoría de la formación. Madrid: Narcea.

Latouche, S. (2009). Pequeno Tratado do Decrescimento Sereno. São Paulo: Editora WMF Martin Fontes.

López, A. y Granada, P. (2007). Formación un concepto para la acción curricular. Revista Académica e Institucional de la UCPR, (79), 53-70. Recuperado de https://dialnet.unirioja.es/servlet/articulo?codigo $=4897789$

López, M; Restrepo, L. y López, G. (2013). Resistencia al cambio en organizaciones modernas. Scientia et Technica, 18(1), 149-157. Recuperado de https://www.redalyc.org/pdf/849/84927487022.pdf

Lucio, R. (2019). Violencia simbólica y formación docente transformadora: P. Freire y la pedagogía del oprimido. En R. Torres y D. Lozano (Eds.) La formación de docentes en América Latina. Perspectivas, enfoques y concepciones criticas (pp. 225-240). México: CLACSO.

Ministerio de Educación Nacional de Colombia [MEN]. (2002). Ley № 715, Decreto №1278. Vinculación, administración y formación de los docentes y directivos docentes en los establecimientos educativos estatales. Bogotá, Colombia: MEN.

Pérez, Y. y Coutín, A. (2005). La gestión del conocimiento: un nuevo enfoque en la gestión empresarial. Acimed, 13(6), 31-40. Recuperado de http://scielo.sld.cu/pdf/aci/v13n6/aci040605.pdf

Sanabria, R. (2017). La educación del ser humano: un reto permanente. Caracas: Universidad Metropolitana. Recuperado de https://n9.cl/03btv

Sánchez, M.; y Galiano, A. (2019). Desarrollo de un equipo docente en la coordinación del profesorado en el ámbito universitario. Revista de Educación Alteridad, 14(1), 98-108. Recuperado de https://n9.cl/y5ydh

Valencia, F. (2016) Hacia la renovación de la formación de los docentes en Colombia: ruta tradicional y ruta polivalente. Pedagogía y Saberes, (45), 11-22. Recuperado de http://www.scielo.org.co/pdf/pys/n45/n45a02.p $\mathrm{df}$

Yang, Z. (2016). La obsolescencia programada (tesis de pregrado). Universidad del País Vasco, Bilbao, España. Recuperado de https://addi.ehu.es/bitstream/handle/10810/19046/YANG_TFG.pdf 